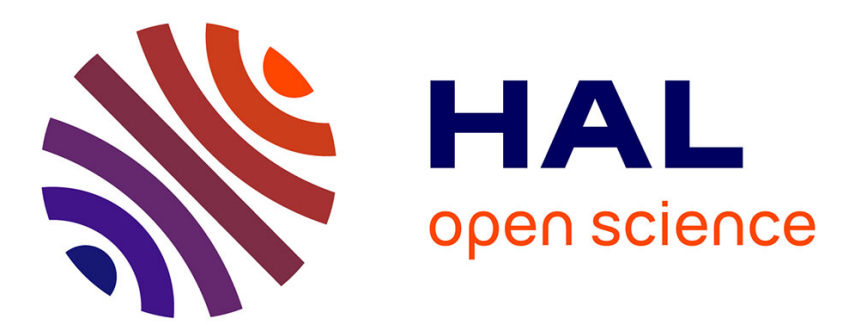

\title{
Une nouvelle technique d'identification de la diffusivité thermique pour la méthode "flash"
}

\author{
A. Degiovanni, M. Laurent
}

\section{To cite this version:}

A. Degiovanni, M. Laurent. Une nouvelle technique d'identification de la diffusivité thermique pour la méthode "flash". Revue de Physique Appliquée, 1986, 21 (3), pp.229-237. 10.1051/rphysap:01986002103022900 . jpa-00245435

\section{HAL Id: jpa-00245435 https://hal.science/jpa-00245435}

Submitted on 1 Jan 1986

HAL is a multi-disciplinary open access archive for the deposit and dissemination of scientific research documents, whether they are published or not. The documents may come from teaching and research institutions in France or abroad, or from public or private research centers.
L'archive ouverte pluridisciplinaire HAL, est destinée au dépôt et à la diffusion de documents scientifiques de niveau recherche, publiés ou non, émanant des établissements d'enseignement et de recherche français ou étrangers, des laboratoires publics ou privés. 
Classification

Physics Abstracts

$44.10-44.50-66.70-72.15$

\title{
Une nouvelle technique d'identification de la diffusivité thermique pour la méthode « flash »
}

\author{
A. Degiovanni \\ LEMTA, UA 875 CNRS, 54042 Nancy Cedex, France
}

et M. Laurent

Laboratoire de Physique Industrielle, INSA, 69000 Villeurbanne, France

(Reçu le 1er août 1985, révisé le 14 novembre, accepté le 15 novembre 1985)

\begin{abstract}
Résumé. - Une nouvelle identification de la diffusivité thermique est proposée pour la méthode « flash ». Elle est fondée sur l'emploi des moments temporels partiels d'ordre 0 et -1 . Elle présente à la fois l'avantage d'être très simple à mettre en ouvre et de prendre en compte toute la partie significative du thermogramme. Son utilisation pour un dispositif expérimental particulier montre qu'elle est bien adaptée à l'utilisation d'un microordinateur.

Abstract. - A new approach is used to identify the thermal diffusivity of solid, homogeneous isotropic material by computing the partial time moments of order 0 and - 1 . In order to test this method, we apply it to the "flash " method for cylindrical samples. Previous studies shows that the evolution in time of normalized temperature $\left(T / T_{\max }\right.$ versus $\left.t\right)$ depends in first approximation only on two parameters : a Biot number $(H)$ which takes in account the heat exchange with the surrounding; a characteristic time $\left(\tau_{0}\right)$. The aim of this study is to identify the two parameters $H$ and $\tau_{0}$ with accuracy and simplicity.
\end{abstract}

La méthode " flash " proposée par J. Parker [1] est actuellement l'une des méthodes de détermination des propriétés thermophysiques des matériaux les plus utilisées. Sa mise en ouvre expérimentale est particulièrement simple, et, son domaine d'application est très étendu aussi bien en température, qu'en types de matériaux : isolants, conducteurs, solides, liquides, granulaires, etc...

Cette méthode est à ranger dans la classe des méthodes impulsionnelles, dont le principe consiste, dans un système thermique, à introduire une perturbation plus ou moins localisée dans le temps et dans l'espace et à relever en un ou plusieurs endroits les évolutions de température en fonction du temps (thermogrammes).

A partir de ces thermogrammes on déterminera une ou plusieurs caractéristiques du système.

Dans le cas de la méthode "flash " le système thermique est un échantillon cylindrique qui reçoit sur sa face avant un bref flux de chaleur fourni par un tube à éclat, un laser ou un faisceau d'électrons, et l'évolution de la température est relevée sur sa face arrière ; à partir de ce thermogramme on détermine la diffusivité thermique du matériau; la principale difficulté étant la prise en compte des échanges avec le milieu extérieur.

De nombreuses techniques ont été proposées pour déterminer la diffusivité thermique du matériau par identification du thermogramme expérimental avec un thermogramme théorique issu d'un modèle, nous en citerons deux qui donnent des résultats satisfaisants. La première, développée par A. Degiovanni [2-4] très simple n'utilise que quelques points du thermogramme; la seconde, proposée par D. Balageas [5], prend en compte toute la partie significative du thermogramme mais est en revanche plus complexe à mettre en ouvre.

Nous présentons ici une nouvelle technique qui réunit les avantages des deux précédentes à savoir simplicité de mise en ouvre et utilisation de toute la partie significative du thermogramme. Elle est fondée sur l'identification des moments temporels partiels d'ordre 0 et -1 issus du thermogramme expérimental et du modèle. Cette identification nous permet simultanément de tenir compte des échanges avec le milieu 
environnant et de déterminer la diffusivité thermique du matériau.

Ensuite nous montrons comment cette technique peut être très facilement utilisée dans un dispositif expérimental géré par un micro-ordinateur.

\section{Modèle théorique.}

Nous avons montré $[6,7]$ précédemment que, moyennant certaines précautions concernant les niveaux de variation des températures, on pouvait utiliser un modèle linéaire pour déterminer la diffusivité thermique dans le cas où la conductivité et la chaleur volumique varient en fonction de la température et dans le cas d'échanges superficiels par rayonnement non linéaires.

Nous utiliserons donc un modèle linéaire. Il est important de préserver toute sa simplicité à la méthode " flash ", aussi, l'impulsion thermique doit être suffisamment brève pour être assimilée à un dirac de flux et uniformément répartie sur la face avant de l'échantillon, ceci afin d'éviter de connaître et de tenir compte d'une représentation spatio-temporelle de l'impulsion.

Les transferts de chaleur dans un échantillon (Fig. 1) cylindrique en matériau homogène et isotrope sont alors décrits par le système d'équation suivant :

$$
\begin{array}{rlrl} 
& \frac{\partial^{2} T}{\partial x^{2}}+\frac{1}{r} \frac{\partial T}{\partial r}+\frac{\partial^{2} T}{\partial r^{2}}=\frac{1}{a} \frac{\partial T}{\partial t} \\
\text { en } \quad x=0 \quad \lambda \frac{\partial T}{\partial x} & =h_{1}\left(T-T_{0}\right)-Q \delta(0) \\
\text { en } \quad x=e & -\lambda \frac{\partial T}{\partial x}=h_{2}\left(T-T_{0}\right) \\
\text { en } x & =R \quad-\lambda \frac{\partial T}{\partial r}=h_{3}\left(T-T_{0}\right) \\
\text { à } \quad t & =0 & T & =T_{0}
\end{array}
$$

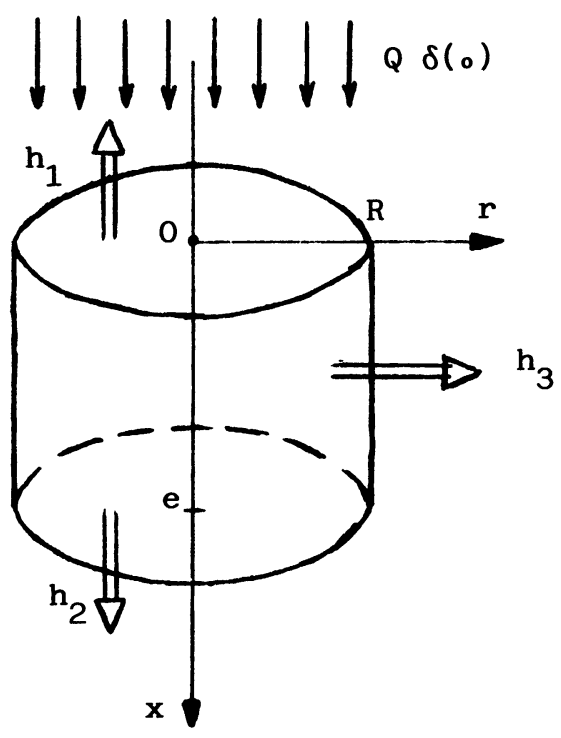

Fig. 1. - Principe de la modélisation.

[Analytical model.] où $T:$ température; $t:$ temps; $\lambda:$ conductivité; $a$ : diffusivité ; $h_{1}, h_{2}, h_{3}$ : les coefficients d'échanges superficiels sur les faces de l'échantillon et $Q \delta(0)$ : énergie par unité de surface de l'impulsion infiniment brève uniformément répartie sur la face $x=0$.

La solution peut être obtenue par séparation des variables [2] soit :

$$
\begin{aligned}
\theta^{*}\left(x^{*}, r^{*}, t^{*}\right)=\sum_{n=1}^{\infty} \sum_{p=1}^{\infty} A_{n p} F_{n}\left(x^{*}\right) G_{p}\left(r^{*}\right) \times & \\
& \times \exp \left(-v_{n p} t^{*}\right)
\end{aligned}
$$

avec :

$$
\begin{gathered}
A_{n p}=\frac{4 \alpha_{n}^{2}\left(\alpha_{n}^{2}+H_{2}^{2}\right)}{\left(\alpha_{n}^{2}+H_{1}^{2}\right)\left(\alpha_{n}^{2}+H_{2}^{2}\right)+\left(H_{1}+H_{2}\right)\left(\alpha_{n}^{2}+H_{1} H_{2}\right)} \times \\
\times \frac{H_{3} R^{*}}{\left(H_{3}^{2} R^{* 2}+\gamma_{p}^{2}\right) J_{0}\left(\gamma_{p}\right)} \\
F_{n}\left(x^{*}\right)=\cos \alpha_{n} x^{*}+\frac{H_{1}}{\alpha_{n}} \sin \alpha_{n} x^{*} \\
G_{p}\left(r^{*}\right)=J_{0}\left(\gamma_{p} r^{*} / R^{*}\right) \\
v_{n p}=\alpha_{n}^{2}+\gamma_{n}^{2} / R^{* 2}
\end{gathered}
$$

où les $\alpha_{n}$ et $\gamma_{p}$ sont solutions respectivement des équations transcendantes :

$$
\begin{aligned}
\left(\alpha^{2}-H_{1} H_{2}\right) \operatorname{tg} \alpha & =\left(H_{1}+H_{2}\right) \alpha \\
\gamma J_{1}(\gamma) & =H_{3} R^{*} J_{0}(\gamma) .
\end{aligned}
$$

Les variables réduites sont définies par :

$$
\begin{aligned}
x^{*} & =x / e ; \quad r^{*}=r / e ; \quad R^{*}=R / e \\
t^{*} & =a t / e^{2} \text { (nombre de Fourier) } ; \theta^{*}=\rho_{c} e\left(T-T_{0}\right) / Q \\
H_{n} & =h_{n} e / \lambda \text { (nombre de Biot) avec } n \text { variant de } 1 \text { à } 3 .
\end{aligned}
$$

Le thermogramme étant relevé en un point de la face $x=e\left(x^{*}=1\right)$ la température en ce point apparaît sous la forme :

$$
\begin{aligned}
\theta^{*}\left(1, r^{*}, t^{*}\right)=\sum_{n=1}^{\infty} \sum_{p=1}^{\infty} f\left(H_{1}, H_{2}, H_{3}, r^{*}, R^{*}\right) \times \\
\times \exp \left(-v_{n p} t^{*}\right) .
\end{aligned}
$$

Cette relation dépend alors de cinq paramètres :

$$
H_{1}, H_{2}, H_{3}, r^{*} \text { et } R^{*} \text {. }
$$

Les deux paramètres géométriques pourraient être mesurés. En pratique s'il n'y a pas de difficulté pour $R^{*}$, ce n'est pas le cas pour $r^{*}$ qui représente la position $\mathrm{du}$ thermomètre et qui peut ne pas être connue avec précision.

En fait, on ne peut comparer directement les thermogrammes expérimentaux avec les courbes théoriques, l'énergie absorbée par l'échantillon n'étant généralement pas connue; on est donc amené à comparer des courbes normalisées calculées à partir du modèle $\theta^{*} / \theta_{\max }^{*}=f^{*}\left(t^{*}\right)$ (où $\theta_{\max }^{*}$ est la température réduite 
maximale) avec des thermogrammes expérimentaux normalisés $\left(T-T_{0}\right) /\left(T_{\max }-T_{0}\right)=f(t)$ (où $T_{\max }$ est la température maximale atteinte).

Comme nous l'avons déjà montré [2], il n'est pas possible d'identifier les cinq paramètres à partir d'un seul thermogramme.

Aussi nous allons choisir des valeurs extrêmes pour les nombres de Biot $H_{1}, H_{2}, H_{3}$ et des valeurs particulières pour les paramètres géométriques. Nous montrerons ensuite l'importance de ces conditions sur la détermination de la diffusivité.

Nous choisissons les cinq cas suivants :

$$
\begin{aligned}
& -\mathrm{n}^{\circ} 1: H_{1}=H_{2}=H ; \quad H_{3}=0 \\
& -\mathrm{n}^{\circ} 2: H_{1}=H ; \quad H_{2}=H_{3}=0 \\
& \text { - no } 3: H_{2}=H ; \quad H_{1}=H_{3}=0 \\
& -\mathrm{n}^{\mathrm{o}} 4: H_{3}=H ; \quad H_{1}=H_{2}=0
\end{aligned}
$$$$
\left(R^{*}=1 \text { et } r^{*}=0\right)
$$

et le cas le plus réaliste (coefficient d'échange identique sur toutes les faces)

$$
\text { - no } 5: H_{1}=H_{2}=H_{3}=H
$$

avec $R^{*}=0,1 ; 0,5 ; 1 ; 1,5 ; 2 ; 4 ; \infty$

et $\quad r^{*}=0 ; 0,5 ; 1$.

Dans ces cas, les thermogrammes normalisés $f^{*}\left(t^{*}\right)$ dépendent d'un seul paramètre : le nombre de Biot $H$; le temps $t$ se déduisant du temps réduit $t^{*}$ à partir d'un temps caractéristique $\tau_{0}=e^{2} / a$, soit en fait deux paramètres à déterminer à partir du thermogramme expérimental : $H$ et $\tau_{0}$.

On pourrait utiliser des méthodes générales d'identification (par minimisation d'une fonction des paramètres à identifier), mais elles sont très mal adaptées à ce type de problème, les deux paramètres agissant de façon totalement différentes sur les thermogrammes; l'un $\left(\tau_{0}\right)$ intervient uniquement dans les constantes de temps, l'autre $(H)$ principalement dans les coefficients d'amplitudes.

\section{Méthode d'identification.}

Nous avons adapté à nos thermogrammes la méthode des moments temporels souvent employée en identification de processus et développée en particulier par J. C. Trigeassou [8, 9]. Nous définissons les moments temporels partiels de la façon suivante :

$$
\begin{aligned}
& m_{i}^{*}=\int_{t_{\alpha}^{*}}^{t_{\beta}^{*}} t^{* i} f^{*}\left(t^{*}\right) \mathrm{d} t^{*} \\
& m_{i}=\int_{t_{\alpha}}^{t_{\beta}} t^{i} f(t) \mathrm{d} t
\end{aligned}
$$

avec $i$ entier positif ou négatif

$t_{\alpha}^{*}$ et $t_{\beta}^{*}$ sont des temps réduits correspondant respectivement à

$$
f^{*}\left(t^{*}\right)=\alpha \quad \text { et } \quad f^{*}\left(t^{*}\right)=\beta .
$$

De même $t_{\alpha}$ et $t_{\beta}$ sont des temps correspondant respectivement à

$$
f(t)=\alpha \quad \text { et } \quad f(t)=\beta .
$$

Le processus dépendant de deux paramètres, il nous faut au minimum deux moments pour les identifier, en gardant à l'esprit que l'on cherche à mesurer avec précision $\tau_{0}$ et non $H$. Nous avons choisi l'ordre -1 et 0 , ce qui permet de privilégier les temps courts pour lesquels l'influence de la diffusivité est prépondérante par rapport à celle des pertes.

Le choix des limites d'intégrations $t_{\alpha}$ et $t_{\beta}$ est moins critique néanmoins, $t_{\beta}$ doit être inférieur à $t_{\max }$ pour les mêmes raisons que précédemment. L'incertitude relative sur la diffusivité pour une erreur absolue constante sur la température (correspondant au bruit de mesure dépendant des conditions expérimentales) a été étudiée par D. L. Balageas [5.].

Cette étude nous permet de choisir $t_{\alpha}$ et $t_{\beta}$ pour minimiser l'incertitude, la courbe (2) en montre l'évolution pour le cas idéal adiabatique en fonction de la température normalisée $\theta^{*} / \theta_{\max }^{*}$ et du temps réduit $t^{*}$; elle présente un minimum pour $t^{*} \simeq 0,1\left(\theta^{*} / \theta_{\max }^{*} \simeq\right.$ $0,35)$ et une évolution assez plate pour $\theta^{*} / \theta_{\max }^{*}$ compris entre 0,1 et $0,8:$ ce sont les valeurs que nous avons choisies respectivement pour $\alpha$ et $\beta$.

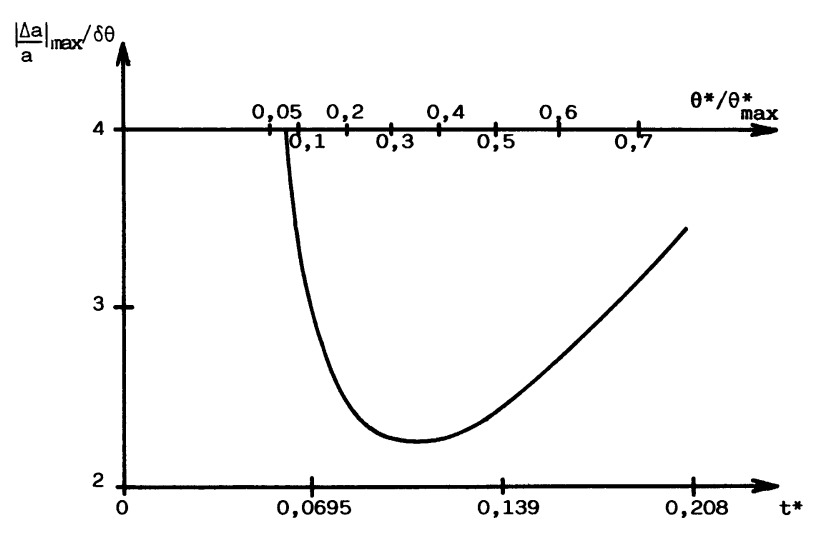

Fig. 2. - Evolution de la sensibilité de la mesure de la diffusivité pour une erreur absolue constante sur la température de la face arrière.

[Precision evolution of diffusivity measurement with constant absolute error on temperature of back face.]

Les moments théoriques réduits sont fonctions seulement de $H$ :

$$
\begin{gathered}
m_{0}^{*}(H)=\int_{t^{*}, 1}^{t_{0,8}^{*}} f^{*}\left(t^{*}\right) \mathrm{d} t^{*} \\
m_{-1}^{*}(H)=\int_{t_{0,1}^{*}}^{t_{0,8}^{*}} \frac{f^{*}\left(t^{*}\right)}{t^{*}} \mathrm{~d} t^{*}
\end{gathered}
$$


et pour les moments expérimentaux $m_{0}$ est fonction de $H$ et $\tau_{0}$ et $m_{-1}$ est seulement fonction de $H$ :

$$
\begin{gathered}
m_{0}\left(H, \tau_{0}\right)=\int_{t_{0,1}}^{t_{0,8}} f(t) \mathrm{d} t \\
m_{-1}(H)=\int_{t_{0,1}}^{t_{0,8}} \frac{f(t)}{t} \mathrm{~d} t .
\end{gathered}
$$

De façon générale, $f(t)$ peut s'écrire sous la forme de série infinie :

$$
f(t)=\sum_{n=1}^{\infty} A_{n} \exp \left(-\alpha_{n}^{2} t / \tau_{0}\right)
$$

d'où l'expression des moments :

$$
m_{i}=\int_{t_{\alpha}}^{t_{\beta}} t^{i} \sum_{n=1}^{\infty} A_{n} \exp \left(-\alpha_{n}^{2} t / \tau_{0}\right) \mathrm{d} t
$$

avec $t^{*}=t / \tau_{0}$ et $i \geqslant-1$

$$
m_{i}=\int_{t_{\alpha}^{*}}^{t_{\beta}^{*}}\left(\tau_{0} t^{*}\right)^{i} \sum_{n=1}^{\infty} A_{n} \exp \left(-\alpha_{n}^{2} t^{*}\right) \tau_{0} \mathrm{~d} t^{*}
$$

soit :

$$
m_{i}=\left(\tau_{0}\right)^{i+1} m_{i}^{*} \text { pour } i \geqslant-1
$$

donc pour $i=-1, m_{-1}=m_{-1}^{*}$ et $m_{-1}$ n'est pas fonction de $\tau_{0}$. Par conséquent indépendant de la diffusivité il caractérise donc les fuites thermiques de l'échantillon.

Nous calculons théoriquement $m_{0}^{*}$ et $m_{-1}^{*}$ en fonction de $H$ en utilisant l'un des modèles thermocinétiques définis précédemment. En éliminant $H$, nous obtenons la fonction d'identification :

$$
m_{0}^{*}=\mathcal{F}\left(m_{-1}^{*}\right) .
$$

A partir du thermogramme expérimental, nous déterminons $m_{0}$ et $m_{-1}$ (Fig. 3). Des relations entre moments théoriques et expérimentaux,

$$
m_{-1}=m_{-1}^{*} \quad \text { et } \quad m_{0}=\tau_{0} m_{0}^{*} .
$$

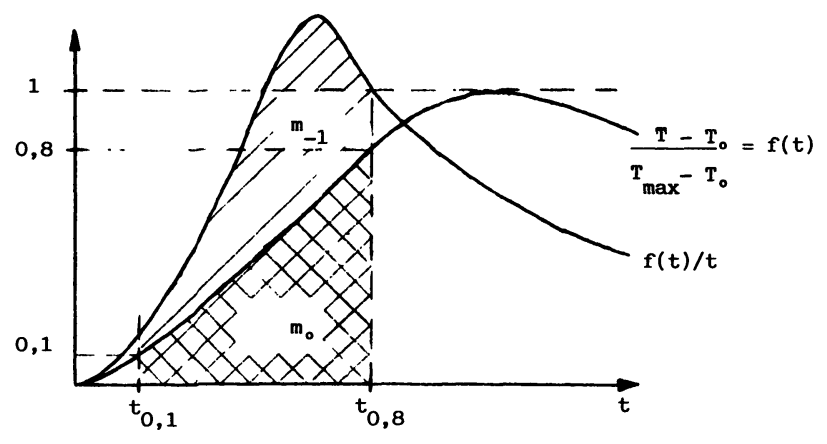

Fig. 3. - Thermogramme.

[Temperature versus time curve.]
Nous obtenons :

$$
\tau_{0}=m_{0} / m_{0}^{*}=m_{0} / \mathcal{F}\left(m_{-1}^{*}\right)=m_{0} / \mathcal{F}\left(m_{-1}\right) .
$$

Soit finalement la diffusivite thermique :

$$
a=\frac{e^{2} \mathfrak{F}\left(m_{-1}\right)}{m_{0}} .
$$

Dans le cas d'échantillon adiabatique, $\mathcal{F}\left(m_{-1}\right) \simeq$ 0,0855 ; on retrouve une formule du type Parker [1] :

$$
a=\frac{0,0855 e^{2}}{m_{0}} \text {. }
$$

\begin{tabular}{|c|c|c|c|c|c|c|c|}
\hline \multicolumn{2}{|c|}{$H_{3}=O \quad H_{1}=H_{2}=H$} & \multicolumn{2}{|c|}{$\begin{array}{ll}\mathrm{H}_{3}=\mathrm{H}_{2}=0 & \mathrm{H}_{1}=\mathrm{H} \\
\mathrm{H}_{3}=\mathrm{H}_{1}=0 & \mathrm{H}_{2}=\mathrm{H}\end{array}$} & \multicolumn{2}{|c|}{$\mathrm{H}_{1}=\mathrm{H}_{2}=\mathrm{H}_{3}=\mathrm{H}$} & \multicolumn{2}{|c|}{$H_{1}=H_{2}=0 \quad H_{3}=H$} \\
\hline $\mathrm{m}^{*}$ 。 & $m^{*}-1$ & $m^{*}$ 。 & $m^{*}-1$ & $m^{*}$ 。 & $m^{*}-1$ & $m^{*}$ o & $m^{*}-1$ \\
\hline 0,08548 & 0,5486 & 0,08548 & 0,5486 & 0,08548 & 0,5486 & 0,08548 & 0,5486 \\
\hline 0,08248 & 0,5402 & 0,08400 & 0,5438 & 0,07807 & 0,5246 & 0,08122 & 0,5246 \\
\hline 0,08091 & 0,5341 & 0,08285 & 0,5402 & 0,07356 & 0,5100 & 0,07838 & 0,5251 \\
\hline 0,07648 & 0,5202 & 0,08010 & 0,5316 & 0,06722 & 0,4889 & 0,07244 & 0,5051 \\
\hline 0,07309 & 0,5094 & 0,07789 & 0,5246 & 0,06478 & 0,4806 & 0,06609 & 0,4831 \\
\hline 0,07119 & 0,5033 & 0,07661 & 0,5206 & 0,05909 & 0,4608 & 0,06173 & 0,4674 \\
\hline 0,06391 & 0,4796 & 0,07644 & 0,5199 & 0,05064 & 0,4297 & 0,05847 & 0,4554 \\
\hline 0,05883 & 0,4624 & 0,07152 & 0,5045 & 0,04977 & 0,4265 & 0,05043 & 0,4241 \\
\hline 0,05182 & 0,4379 & 0,06775 & 0,4924 & 0,04034 & 0,3887 & 0,04912 & 0,4188 \\
\hline 0,04511 & 0,4130 & 0,06225 & 0,4744 & 0,03605 & 0,3704 & 0,04315 & 0,3938 \\
\hline 0,04348 & 0,4067 & 0,05669 & 0,4559 & 0,03195 & 0,3518 & 0,03869 & 0,3741 \\
\hline 0,04197 & 0,4008 & 0,05527 & 0,4510 & 0,02529 & 0,3201 & 0,03746 & 0,3685 \\
\hline 0,03656 & 0,3789 & 0,05398 & 0,4466 & 0,02484 & 0,3178 & 0,03370 & 0,3514 \\
\hline 0,03303 & 0,3639 & 0,04912 & 0,4298 & 0,02038 & 0,2954 & 0,03278 & 0,3470 \\
\hline 0,02973 & 0,3491 & 0,04583 & 0,4181 & 0,01803 & 0,2833 & 0,03150 & 0,3412 \\
\hline 0,02099 & 0,3085 & 0,04266 & 0,4068 & 0,01712 & 0,2794 & 0,03113 & 0,3394 \\
\hline \multirow[t]{2}{*}{0,01680} & 0,2897 & 0,03398 & 0,3763 & 0,01516 & 0,2709 & 0,03041 & 0,3362 \\
\hline & & 0,02987 & 0,3646 & 0,01477 & 0,2691 & 0,03034 & 0,3359 \\
\hline
\end{tabular}

\section{Fonction d'identification.}

Dans le tableau I et sur la figure 4, nous avons porté la fonction d'identification calculée pour les quatre premiers cas et le cas $n^{\circ} 5$ avec $R^{*}=1$ et $r^{*}=0$, pour des nombres de Biot inférieurs à 25 .

Tableau I. - Influence de la répartition des échanges dans le cas $r^{*}=0$ et $R^{*}=1$.

[Influence of heat loses distribution in case of $r^{*}=0$ et $R^{*}=1$.]

Il est à noter que les cas $n^{\circ} 2$ et $n^{\circ} 3$ donnent analytiquement les mêmes résultats.

La figure 4 montre que lorsque $H$ reste inférieur à 1 , les fonctions d'identification restent très proches les unes des autres. Par contre, pour $\boldsymbol{H}$ grand, elles diffèrent selon les cas étudiés; il sera donc nécessaire de connaître le type de répartition des fuites thermiques (par une analyse des caractéristiques du montage vis-à-vis des échanges échantillon-milieu extérieur). En général il n'y a aucune raison particulière de choisir des coefficients d'échange différents sur chaque face, on utilisera alors le cas no $5\left(H_{1}=H_{2}=H_{3}=H\right)$. 


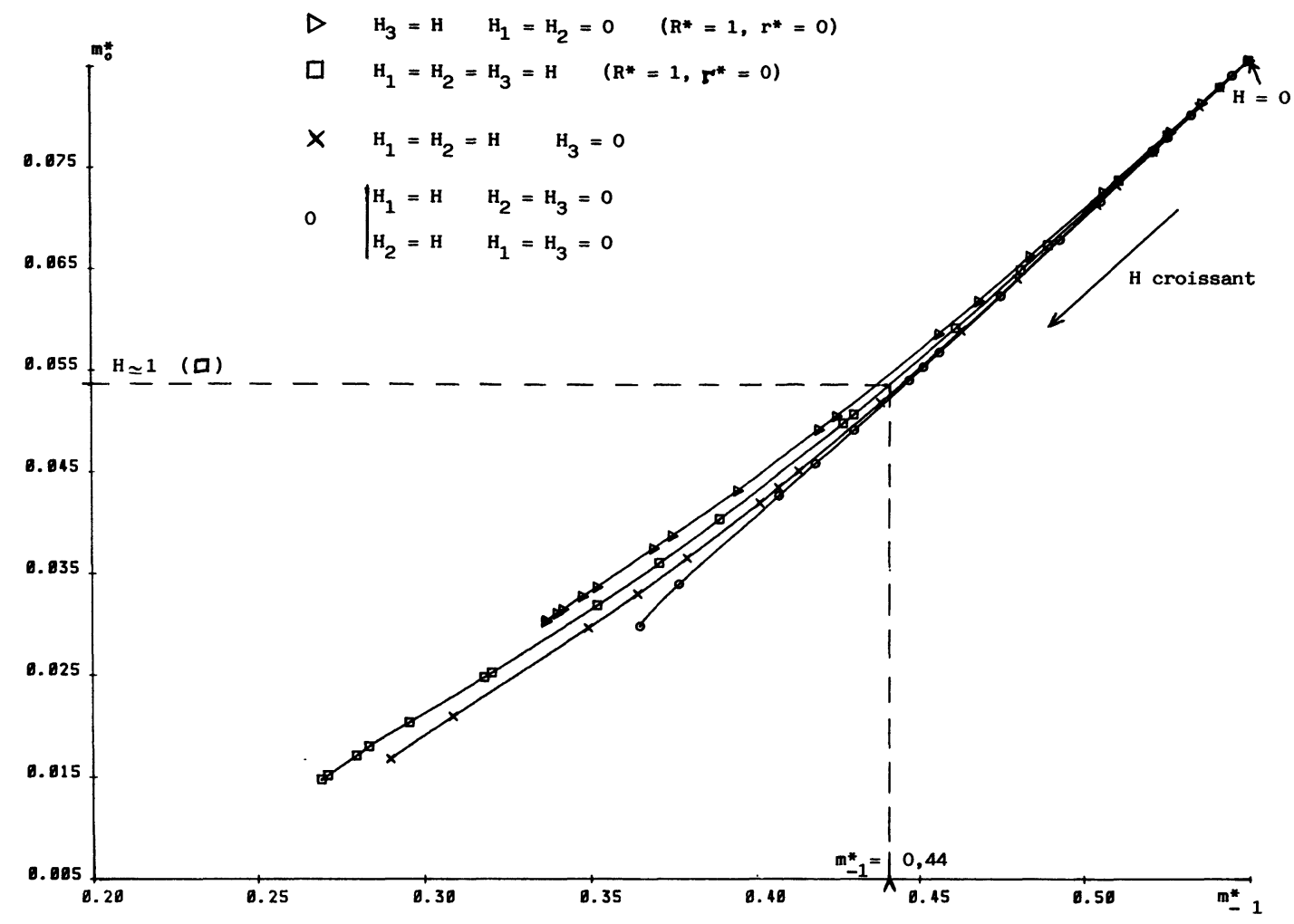

Fig. 4. - Fonction d'identification (pour différentes répartitions des échanges).

[Identification function (influence of heat loses distribution).]

Le tableau II et la figure 5 montrent l'influence des caractéristiques géométriques $\left(R^{*}\right)$ sur la fonction d'identification $\left(R^{*}\right.$ varie de 0,1 à l'infini). Nous constatons aucune influence lorsque $m_{-1}$ est supérieur à 0,44 (ce qui correspond à la plupart des cas pratiques pour lesquels $H$ est inférieur à 1 ). Il existe en revanche une très faible dispersion pour les grandes valeurs du coefficient d'échange. Pour des mesures à très haute température sur des isolants il sera donc nécessaire d'utiliser la fonction d'identification correspondant à la valeur $R^{*}$ de l'échantillon étudié.

En ce qui concerne les valeurs de $r^{*}$ (Tableau III, Fig. 6), elles n'ont aucune influence significative sur la fonction d'identification tant que $r^{*}<0,5$ (ce point

Tableau II. - Influence du rapport rayon sur épaisseur de l'échantillon dans le cas $H_{1}=H_{2}=H_{3}=H_{\text {et }} r^{*}=0$.

[Influence of radius/thickness ratio in case of $H_{1}=H_{2}=H_{3}=H$ and $r^{*}=0$.]

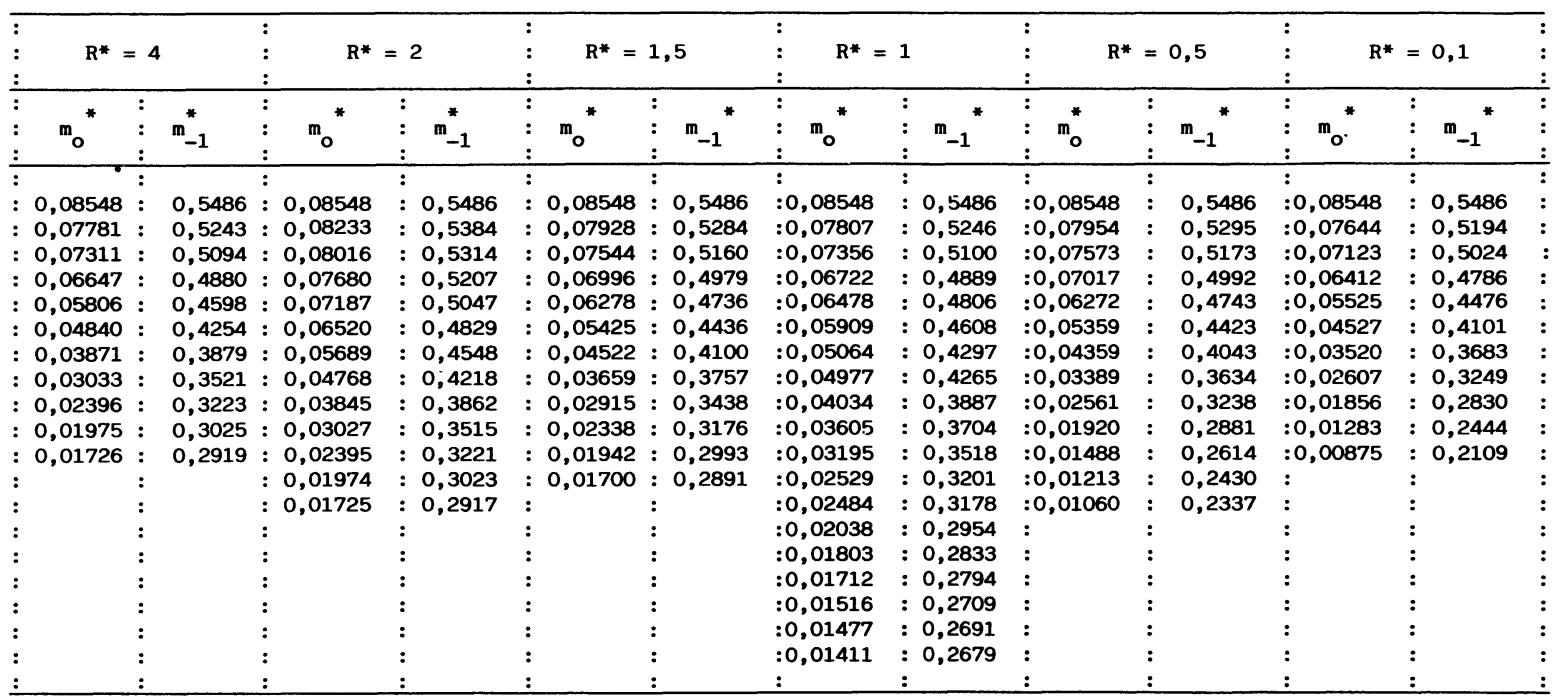




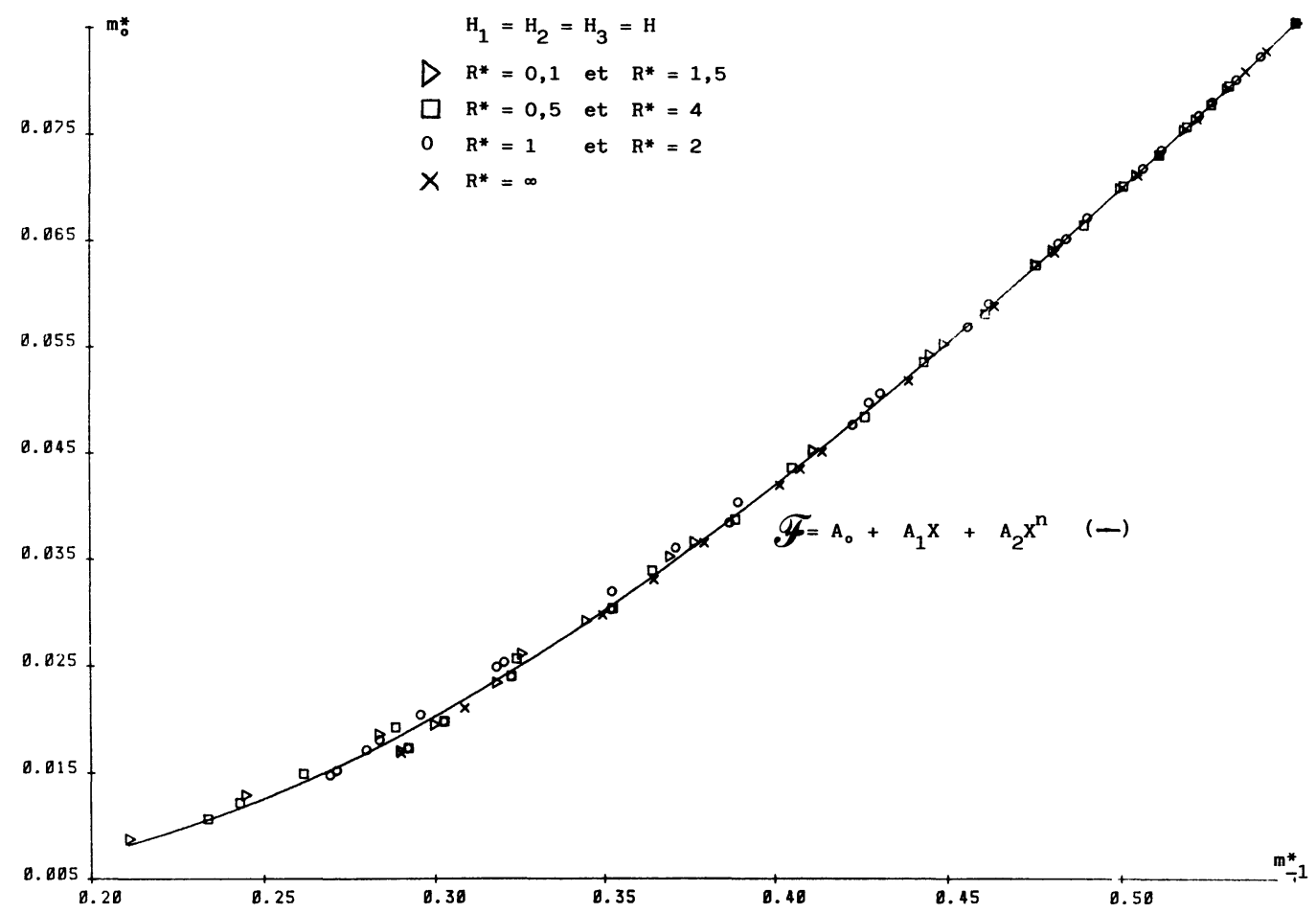

Fig. 5. - Fonction d'identification (pour différentes valeurs de $R^{*}$ ).

[Identification function (influence of radius/thickness ratio).]

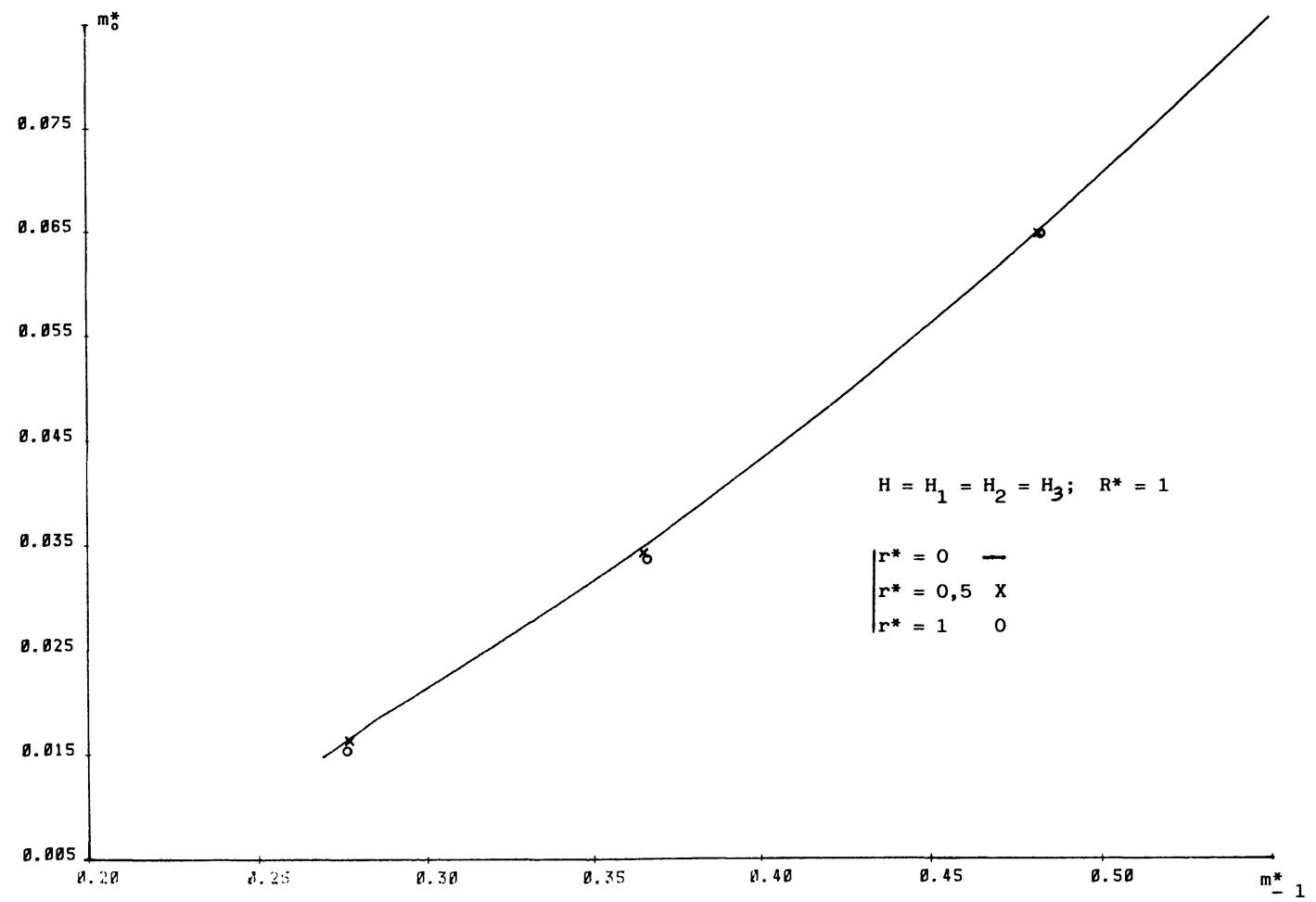

Fig. 6. - Fonction d'identification (pour différentes valeurs de $r^{*}$ ).

[Identification function (influence of $r^{*}$ ).] 
Tableau III. - Influence du point de mesure sur les moments dans le cas $H_{1}=H_{2}=H_{3}=H$ et $R^{*}=1$. [Influence of measurement place in case of $H_{1}=H_{2}=$ $H_{3}=H$ and $R^{*}=1$.]

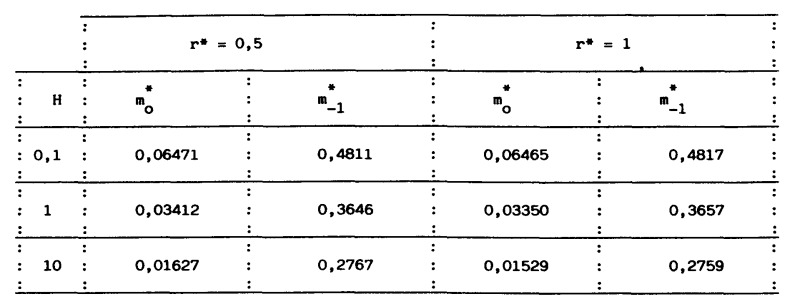

est très important car $r^{*}$ peut ne pas être connu avec précision).

\section{Sensibilité au bruit.}

Nous avons vérifié la sensibilité de notre modèle au bruitage du thermogramme. Les résultats sont donnés pour un bruit aléatoire correspondant à $10 \%$ de $\theta_{\max }^{*}$; le calcul des moments étant effectué en utilisant 50 valeurs de la courbe bruitée, on obtient :

$$
\begin{array}{ll}
H=0,01 & 1 / \tau_{0}=1,014 \text { soit un écart de } 2 \% \\
H=0,2 & 1 / \tau_{0}=1,029 \text { soit un écart de } 3 \% \\
H=2 & 1 / \tau_{0}=0,957 \text { soit un écart de } 4 \%
\end{array}
$$

Les écarts deviennent négligeables si l'on utilise 1000 valeurs pour le calcul des moments ou si le bruit reste inférieur à $1 \%$ de $\theta_{\max }^{*}$.

\section{Utilisation de cette méthode.}

Nous montrons maintenant, comment cette méthode peut être utilisée pour un dispositif expérimental géré par un micro-ordinateur; nous ne ferons qu'une description sommaire du dispositif (Fig. 7) qui a été décrit par ailleurs [2, 3].

L'échantillon est dans une enceinte dont la température peut être régulée entre $-196^{\circ} \mathrm{C}$ et $+100^{\circ} \mathrm{C}$.

L'impulsion thermique est fournie par un tube à éclat.

Le signal correspondant est amplifié, et son « offset » compensé.

L'acquisition est effectuée à l'aide d'un microordinateur qui pilote complètement le dispositif et traite les thermogrammes.

La cellule d'essai est constituée d'une enceinte étanche double paroi présentant une fenêtre dans sa partie supérieure. Au-dessus de cette fenêtre se trouve un écran double paroi amovible commandé par un vérin.

Cet écran est enlevé et remplacé par le tube à éclat lorsque le "flash » est "délivré ». Dans les doubles parois un fluide régulé en température circule. Pour les températures les plus basses l'enceinte est plongée dans de l'azote liquide.

La température du bloc de cuivre est mesurée avec une « résistance » de platine.

Les variations de température de la face arrière de l'échantillon sont relevées à l'aide de détecteurs thermoélectriques semi-conducteurs $\left(\mathrm{Bi}_{2} \mathrm{Te}_{3}\right)$ à pouvoir thermoélectrique élevé $360 \mu \mathrm{V} / \mathrm{K}$ à $20^{\circ} \mathrm{C}$. La jonction du thermocouple est assurée par l'échantillon lui-même s'il est conducteur électrique ou par une mince couche conductrice déposée s'il est isolant.

L'échantillon poussé par les détecteurs de température repose en trois points sur l'autre face. Si le matériau est transparent ou réflecteur la face recevant le flash est recouverte d'un dépôt absorbant (noir).

Un micro-ordinateur pilote l'alimentation du flash

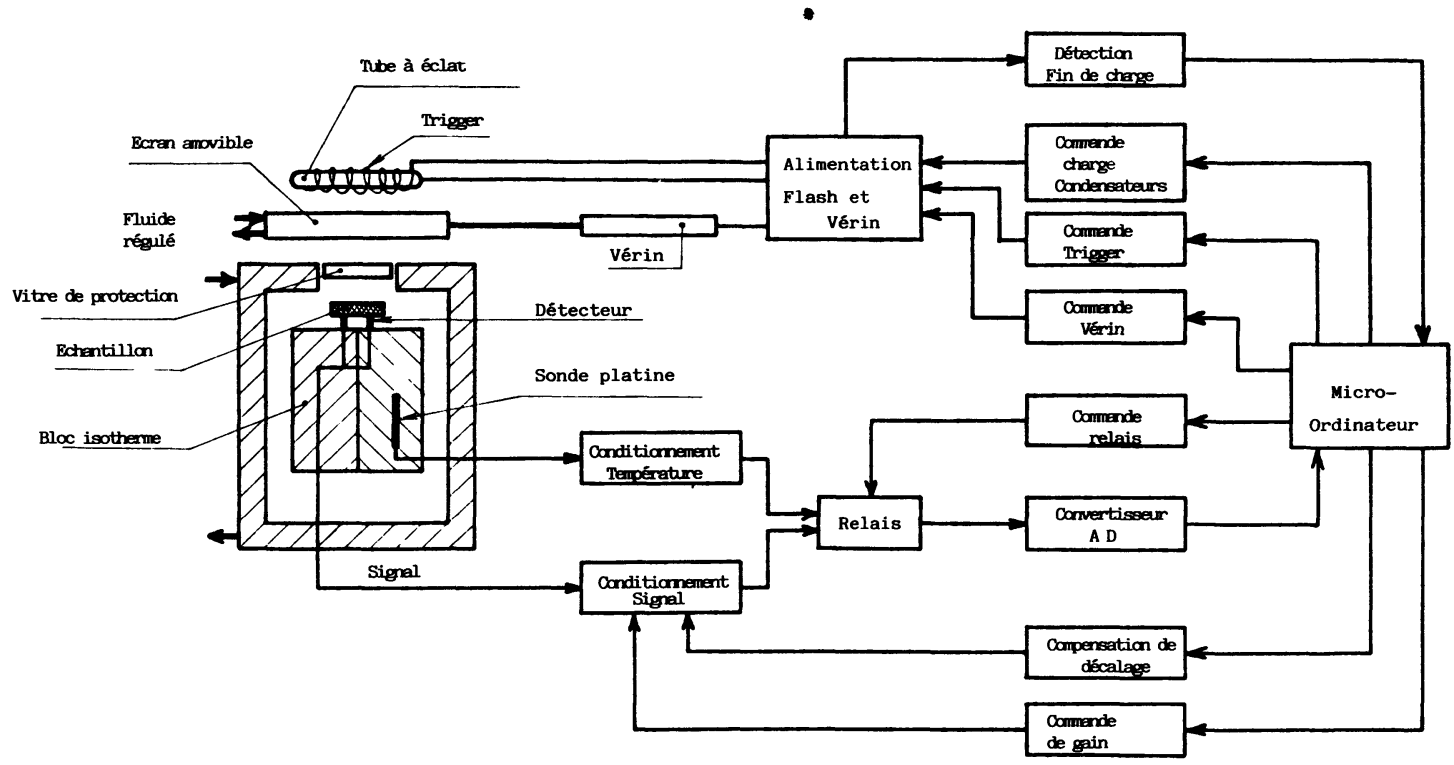

Fig. 7. - Schéma de principe de l'installation.

[Schematic diagram of experimental apparatus.] 
et le vérin. Il relève la température indiquée par la résistance de platine et le thermogramme expérimental.

La force électromotrice délivrée par les détecteurs de température correspond à l'écart entre la face arrière de l'échantillon et les blocs en cuivre. Cet écart est la somme d'un décalage permanent et de la variation due à l'impulsion thermique. Seule cette variation sert à la détermination de la diffusivité. La tension de décalage est éliminée par un asservissement.

Avant la mesure, le signal issu des détecteurs thermoélectriques est amplifié puis converti numériquement et intégré par le microordinateur. Cette valeur est convertie analogiquement et soustraite au signal partiellement amplifié des détecteurs, la valeur intégrée croît jusqu'à l'équilibre de l'asservissement, c'est-à-dire jusqu'à ce que la tension convertie numériquement soit nulle. Ensuite la valeur réinjectée sera maintenue constante et égale à la dernière valeur obtenue et le décalage sera compensé. Avant la mesure, l'acquisition du signal est faite pour compenser, numériquement, une éventuelle dérive thermique. Ensuite, les tubes à éclats sont mis en place, le flash déclenché, enfin l'écran remis à sa position initiale. L'acquisition du thermogramme s'effectue avec deux fréquences d'échantillonnage, une grande pour le calcul des moments et une plus faible pour la recherche du maximum. Le gain de l'amplificateur, le nombre des échantillons et leur fréquence sont réglables.

Lorsque l'acquisition est terminée la valeur réinjectée par l'asservissement est annulée ce qui permet de connaître l'écart de température entre l'échantillon et les blocs de cuivre (source froide du couple). La température de l'échantillon peut alors être calculée compte tenu de cet écart.

C'est cette température qui sera la température d'essai. Nous avons, en effet, montré précédemment $[6,7]$ que c'est une température voisine de la température du maximum qui devait être prise en cas de non-linéarités.

Les valeurs sont compensées de la dérive relevée avant la mesure. La température maximale $\left(T_{\max }-T_{0}\right)$ déterminée après un lissage autour du maximum, le thermogramme est approché par un polynôme du 5e degré entre $0,09 T_{\max }$ et $0,81 T_{\max }$, le calcul des moments s'effectuant sur ce polynôme.

Le calcul de la diffusivité utilise une forme polynomiale de la fonction d'identification (forme utilisable pour $m_{-1}>0,27$ )

avec

$$
\mathcal{F}=A_{0}+A_{1} X+A_{2} X^{n}
$$

$$
\begin{aligned}
A_{0} & =\left(m_{0}^{*}\right)_{H=0} \\
X & =\left(m_{-1}^{*}\right)_{H=0}-m_{-1}^{*}
\end{aligned}
$$

soit

$$
\begin{aligned}
\mathcal{F}=0,08548-0,314 & \left(0,5486-m_{-1}^{*}\right)+ \\
& +0,500\left(0,5486-m_{-1}^{*}\right)^{2,63} .
\end{aligned}
$$

Pour $-m_{-1}>0,44$ la fonction $\mathcal{F}$ peut être représentée par une droite unique indépendante de la répartition des échanges et des caractéristiques géométriques

$$
\mathfrak{F}=-0,0819+0,305 m_{-1}^{*} .
$$

Des mesures ont été faites à température ambiante sur trois échantillons "épais " et isolants. Le tableau IV montre la bonne concordance des résultats obtenus par deux autres méthodes développées par A. Degiovanni [2-4] et D. L. Balageas [5].

Tableau IV. - Mesure de la diffusivité d'échantillons de plâtre et craie.

[Thermal diffusivity of plaster and chalk samples.]

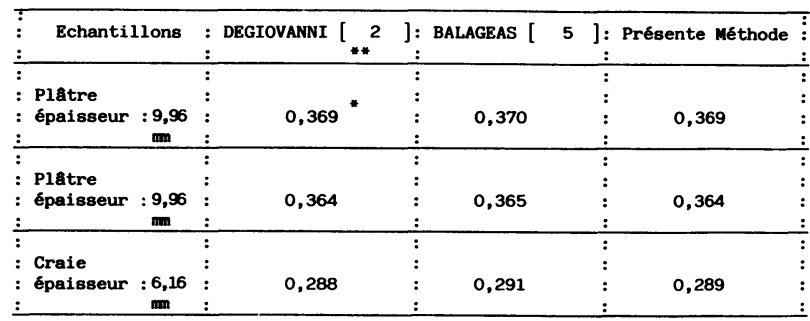

* Diffusivité en $\mathrm{m}^{2} / \mathrm{s}$

* Moyenne des trois valeurs fournies par cette méthode suivant les points de la courbe utilisés.

\section{Conclusion.}

L'avantage de cette nouvelle approche est de conserver à la méthode impulsionnelle sa simplicité d'utilisation, tout en l'adaptant aux techniques modernes d'acquisition et de traitement des données sur microordinateur.

L'identification nécessite seulement la mise en mémoire de la fonction d'identification $\mathcal{F}$ et de l'épaisseur de l'échantillon $e$; la recherche de la valeur maximale du thermogramme $\theta_{\max }^{*}$ et le calcul de deux intégrales $m_{0}$ et $m_{-1}$.

Les moments temporels partiels ont été utilisés ici pour identifier la diffusivité; leur emploi peut, de façon générale, être envisagé lors de la mise en œuvre des techniques impulsionnelles pour déterminer les paramètres de systèmes thermiques complexes.

\section{Nomenclature.}

$a$ diffusivité thermique

$e \quad$ épaisseur de l'échantillon

$f^{*}\left(t^{*}\right) \quad$ courbe de température calculée normalisée

$f(t) \quad$ thermogramme expérimental normalisé

$\mathcal{F}$ fonction d'identification

$h_{1}, h_{2}, h_{3} \quad$ coefficients d'échange

$H_{1}, H_{2}, H_{3}$ nombres de Biot associés à $h_{1}, h_{2}, h_{3}$

$m_{i} \quad$ moment d'ordre $i$ 


$\begin{array}{ll}r & \text { variable d'espace radiale } \\ Q \delta(0) & \text { énergie par unité de surface de l'impulsion } \\ & \text { infiniment brève } \\ r^{*} & r / e \\ R & \text { rayon de l'échantillon } \\ R^{*} & R / e \\ t & \text { temps } \\ t^{*} & t / \tau_{0} \\ t_{\alpha} & \text { temps correspondant à } \alpha T_{\max } \\ t_{\alpha}^{*} & \text { temps réduit correspondant à } \alpha \theta_{\max }^{*} \\ T & \text { température } \\ T_{\max } & \text { température maximale }\end{array}$

$T_{0}$
$x$
$x^{*}$
$\alpha_{n}$
$\gamma_{n}$
$\theta^{*}$
$\theta_{\max }^{*}$
$\lambda$
$\rho_{c}$
$\tau_{0}$
$J_{0}$ et $J_{1}$

température d'équilibre variable d'espace axiale $x / e$ solution d'équation transcendante solution d'équation transcendante température réduite température réduite maximale conductivité thermique chaleur volumique $e^{2} / a$ fonctions de Bessel de $1^{\text {re }}$ espèce d'ordre 0 et 1

\section{Bibliographie}

[1] Parker, W. J., Jenkins, R. J., Butler, C. P. and Aввот,,$G$. L. Flash method of determining thermal diffusivity, heat capacity and thermal conductivity. J. Appl. Phys. 32 no 9 (1961) 1679-1684.

[2] Degiovanni, A., Contribution à l'étude de la diffusivité thermique. Thèse de Doctorat d'Etat no 75-19. Lyon, juin 1975.

[3] Degiovanni, A., Diffusivité et méthode flash. Revue Gén. Therm. 185 (1977) 417-442.

[4] Degiovanni, A., Laurent, M. et Prost, R., Mesure automatique de la diffusivité thermique. Revue Phys. Appl. 14 (1979) 927-932.

[5] Balageas, D. L., Nouvelle méthode d'interprétation des thermogrammes pour la détermination de la diffusivité par la méthode impulsionnelle. Revue Phys. Appl. 17 (1982) 227-237.
[6] Deglovanni, A., Solhili, Z. Critères de non-linéarité pour la mesure de la diffusivité thermique par méthode impulsionnelle. Revue Gén. Therm. 272273 (1984) 489-493.

[7] Degiovanni, A., Sinicki, G., Laurent, M., Heat pulse thermal diffusivity measurements. Thermal properties temperature dependence and non-uniformity. Eighteen International Thermal Conductivity Conference Rapid City. South Dakota, 3-5 Oct. 1983.

[8] Trigeassou, J. C., Identification et commande des processus mono-entrée mono-sortie par la méthode des moments. Thèse de 3e Cycle ENSM Nantes 1980.

[9] Trigeassou, J. C., Identification de processus par la méthode des moments temporels. A.M.S.E. Nice 1983. 